\title{
Vitamin D-binding protein prevents vitamin D deficiency and presents vitamin $D$ for its renal activation
}

\author{
Jens P Berg \\ Hormone Laboratory, Aker University Hospital, N-0514 Oslo, Norway; Email: jenspb@ulrik.uio.no
}

Two mouse models in which the vitamin D-binding protein (DBP) or megalin, a DBP receptor, were knocked out have recently confirmed that DBP is essential for normal vitamin $\mathrm{D}$ homeostasis, and have revealed a major pathway for vitamin $\mathrm{D}$ activation $(1,2)$.

DBP is also known as the group-specific component of serum (Gc-globulin). It is a highly polymorphic $58 \mathrm{kDa}$ glycoprotein, which has been used as a serum protein marker in studies of population genetics. Several biological functions have been associated with DBP, which is a member of a gene family including albumin and $\alpha$-fetoprotein. DBP can prevent polymerization of G-actin into F-actin after traumas by binding and sequestering G-actin, and can activate macrophages and enhance chemotaxis. Most importantly, it binds almost all calcidiol (25-hydroxyvitamin $\mathrm{D}_{3}$ ) molecules in the circulation and leaves only $0.003 \%$ of the metabolite in the free form, whereas calcitriol $(1,25-$ dihydroxyvitamin $\mathrm{D}_{3}$ ) and vitamin $\mathrm{D}_{3}$ are bound with an affinity which is approximately ten times lower $\left(4 \times 10^{-7} \mathrm{M}^{-1}\right)$ (3). According to the 'free hormone' hypothesis, the sterols bound to $\mathrm{DBP}$ are a reservoir that can be made available to vitamin $\mathrm{D}$ receptors or enzymes that modify the sterols by dissociation from the binding protein (4). Although sera from many thousands of individuals have been studied, not a single person has been identified without DBP. Consequently, DBP has been regarded as essential to human survival.

However, when Safadi et al. (1) used targeted mutagenesis to generate mice without DBP, normal viability, fertility and fecundity were observed. The mice had normal growth curves and showed no histological or other abnormalities. Analyses of serum revealed that the homozygous knock-out mice $\left(\mathrm{DBP}^{-/-}\right)$had no detectable DBP, whereas the heterozygous mice $\left(\mathrm{DBP}^{+/-}\right)$had approximately two-thirds the DBP levels of the wild type animals $\left(\mathrm{DBP}^{+/+}\right)$. On a standard diet, serum levels of calcidiol and calcitriol in $\mathrm{DBP}^{-/-}$ mice were approximately $10 \%$ and $15 \%$ of the $\mathrm{DBP}^{+/+}$ mice respectively, whereas the heterozygotes had intermediate levels. Calcium and parathyroid hormone (PTH) levels were not significantly different among the genotypes. In this situation, equilibrium was established between the low total levels of extracellular vitamin $\mathrm{D}$ and the activation of the intracellular vitamin $\mathrm{D}$ receptor.
Increasing or decreasing the supply of vitamin D in the diet changed the situation dramatically. On a vitamin D-deficient diet, serum levels of calcidiol and calcitriol decreased to the limit of detection in both $\mathrm{DBP}^{-/-}$and $\mathrm{DBP}^{+/+}$animals, serum calcium remained constant, but PTH levels doubled in $\mathrm{DBP}^{-1-}$ mice compared with $\mathrm{DBP}^{+/+}$mice. Skeletal abnormalities were observed in the $\mathrm{DBP}^{-/-}$mice, with increased osteoblastic activity and undermineralization of the newly synthesized bone matrix as in hypovitaminosis D osteopathy. The hyperparathyroidism induced increased osteolysis. Studies of the serum half-life of calcidiol confirmed that DBP prevented a rapid metabolization and excretion of vitamin D in urine. In the absence of DBP the uptake of cholecalciferol in the liver increased 15-fold and the sterol was preferentially metabolized to watersoluble products that were excreted in the urine. On the other hand, these metabolic pathways protected the $\mathrm{DBP}^{-/-}$animals from the toxic effects of high-level administration of vitamin D. The capacity of the organism to save vitamin D by DBP may be fatal in a situation of vitamin $\mathrm{D}$ excess.

DBP binding to cell surfaces has been reported. This may be a mechanism that facilitates the entry of vitamin D into target cells. DBP may also slow the release of calcitriol and regulate the level of free sterol available. Safadi et al. (1) showed that in the absence of DBP the kinetics of the mRNA expression of a vitamin D-regulated gene, calbindin- $\mathrm{D}_{9 \mathrm{k}}$, were more rapid, whereas the level of expression was the same as in $\mathrm{DBP}^{+/+}$animals. The primary role of DBP in the study by Safadi et al. (1) was related to the sequestration of vitamin D sterols in the circulation. Evolution has clearly been in favour of the conservation of vitamin $\mathrm{D}$ rather than protecting the organism from vitamin $\mathrm{D}$ toxicity.

Nykjaer et al. (2) recently showed that DBP is also involved in other aspects of vitamin D metabolism. In the kidneys calcidiol is converted to the more potent calcitriol by $1 \alpha$-hydroxylation, which is regulated mainly by PTH (5). It has been suggested that calcidiol is absorbed at the basolateral side of the tubules from the small non-protein-bound fraction (3).

In the glomerulus, DBP is filtered into the preurine and is reabsorbed by endocytosis in the proximal tubules. It has been shown that megalin, a multifunctional receptor and a member of the low-density 
lipoprotein receptor superfamily, is expressed on the lumenal surface of the tubular epithelium in the kidneys (6). It was therefore suggested that the protein could be involved in the reabsorption of filtered macromolecules such as DBP. Most of the megalin knock-out mice die of developmental deficiencies in the forebrain and only $1 / 50$ survive to adulthood (7). Nykjaer et al. (2) studied vitamin D metabolism in the surviving mice lacking megalin and revealed severe vitamin D deficiency and bone disease.

No proteins larger than serum albumin (68 kDa) were observed in the urine of megalin-deficient mice, but several low molecular mass proteins were identified. The sequence of the first part of the major $58 \mathrm{kDa}$ protein excreted in the urine was identical to the first part of mature mouse DBP. Both urine and serum DBP bound specifically to megalin with high affinity in the absence or presence of calcidiol bound to DBP. The DBP-megalin complexes were internalized and processed by lysosomal degradation in the tubular cells. It has previously been shown that the $39 \mathrm{kDa}$ receptorassociated protein (RAP) can block megalin activity. Microinfusion of both RAP and DBP into proximal tubules of anesthetized normal rats decreased the absorption of DBP from $75 \%$ to $8 \%$. When radioactively labeled DBP was infused directly into the renal arteries of rats, more than $80 \%$ of the radioactivity was found in the circulation as proteolytic degradation products $1 \mathrm{~h}$ later. Coinjection with RAP reduced the level of degraded DBP in serum to approximately $10 \%$, whereas more than $80 \%$ was excreted in the urine. Repeating the experiments with labeled calcidiol instead of labeled DBP confirmed that loss of DBP in urine is accompanied by a similar loss of calcidiol. The reabsorption of calcidiol-DBP complexes from the glomerular filtrate by megalin seems to be of great importance for vitamin D homeostasis.

To determine whether the reabsorbed calcidiol may function as a substrate for tubular vitamin D hydroxylases, rats were perfused with radioactively labeled calcidiol in complex with DBP, in the absence or presence of RAP, for 30-40 min. In the presence of RAP, the reabsorption of radiolabeled calcidiol was blocked, and no conversion to calcitriol was detected. In the absence of RAP, both radiolabeled calcidiol and calcitriol were identified in the circulation. The results indicated that megalin is necessary for the reabsorption of DBP and calcidiol from the glomerular filtrate, and that reabsorbed calcidiol can serve as a substrate for renal $1 \alpha$-hydroxylase. Histological examination of the bones of megalin knock-out mice revealed defects associated with vitamin D deficiency. Hypovitaminosis $\mathrm{D}$ is associated with hypocalcemia and secondary hyperparathyroidism. It has recently been shown that megalin may attenuate the effects of PTH in the tubular epithelium by mediating the uptake and degradation of urinary PTH (8). Absence of megalin or blocking megalin with RAP would further increase PTH-receptor activation and $1 \alpha$-hydroxylase activity.

Vitamin D homeostasis obviously depends on sterols bound to DBP as a reservoir of vitamin D. However, DBP has also a unique role in the delivery of calcidiol to the renal calcitriol-producing cells. The changes in vitamin D metabolism in mice lacking megalin may explain previous observations of a direct correlation between glomerular filtration rate and plasma calcitriol levels in patients with kidney diseases (9). Nykjaer et al. (2) have shown that DBP is filtered in the glomeruli and reabsorbed from the preurine by high-affinity binding to megalin in the renal tubules. The megalinDBP complexes are degraded in lysosomes in the tubular cells, and calcidiol, which is bound to $1 \%$ of the DBP molecules, is released. This endocytotic process provides substrate for the tubular $1 \alpha$-hydroxylase, which produces calcitriol. The close connection between activation of vitamin D and normal glomerular filtration rate, including tubular reabsorption of macromolecules, illustrates how vulnerable bone formation is to kidney diseases.

\section{References}

1 Safadi FF, Thornton P, Magiera H, Hollis BW, Gentile M, Haddad JG et al. Osteopathy and resistance to vitamin D toxicity in mice null for vitamin D binding protein. Journal of Clinical Investigation 1999 $103239-251$.

2 Nykjaer A, Dragun D, Walther D, Vorum H, Jacobsen C, Herz J et al. An endocytic pathway essential for renal uptake and activation of the steroid 25-(OH)vitamin $\mathrm{D}_{3}$. Cell 199996 507-515.

3 Haddad JG. Plasma vitamin D-binding protein (Gc-globulin): multiple tasks. Journal of Steroid Biochemistry and Molecular Biology 199553 579-582.

4 Mendel CM. The free hormone hypothesis: a physiologically-based mathematical model. Endocrine Reviews 198910 232-274.

5 Takeyama K-i, Kitanaka S, Sato T, Kobori M, Yanagisawa J \& Kato S. 25-Hydroxyvitamin D3 $1 \alpha$-hydroxylase and vitamin D synthesis. Science 1997277 1827-1830.

6 Gliemann J. Receptors of the low density lipoprotein (LDL) receptor family in man. Multiple functions of the large family members via interaction with complex ligands. Biological Chemistry 1998379 951-964.

7 Willnow TE, Hilpert J, Armstrong SA, Rohlmann A, Hammer RE, Burns DK \& Herz J. Defective forebrain development in mice lacking gp330/megalin. Proceedings of the National Academy of Sciences of the USA 199693 8460-8464.

8 Hilpert J, Nykjaer A, Jacobsen C, Wallukat G, Nielsen R, Moestrup SK et al. Megalin antagonizes activation of the parathyroid hormone receptor. Journal of Biological Chemistry $19992745620-5625$.

9 St John A, Thomas MB, Davies CP, Mullan B, Dick I, Hutchison B et al. Determinants of intact parathyroid hormone and free 1,25dihydroxyvitamin D levels in mild and moderate renal failure. Nephron $199261422-427$. 